\title{
Textbook Inspection and Censorship in Korea during the Protectorate Period: A Study of Inspection Copies of Textbooks Compiled by the Young Korean Academy*
}

Kim Soyoung**

\section{Introduction}

The censorship system emerged in modern Korea during the period of Japanese colonial rule as a measure to improve the efficiency of governing. Established at a time when Korea became a Japanese protectorate, the censorship system lasted throughout the colonial period. Therefore it is necessary to examine the censorship system as part of Japan's colonization policy and shed light on the historical and current significance of the censorship system as well as its effects on Korea. In order to understand the role the censorship system played in maintaining and strengthening Japan's colonial rule over Korea, we need to examine the censorship system from the early stages of its establishment during the protectorate period.

Among a series of laws and regulations related to censorship, this paper will focus on the "Regulations on Textbooks," promulgated in September 1908 , and the cases of actual application of the regulations. These regulations on textbook inspection were enacted by the Japanese Resident-

* This work was supported by the National Research Foundation of Korea Grant funded by the Korean Government(NRF-2014S1A5B4037006).

** Research Professor, Center for Korean History, Korea University 
General of Korea along with the Decree Concerning Private Schools as a means to impede the national education of Koreans. This was part of Japan's policy to completely turn Korea into its colony, following the establishment of the Korean Empire as a "protectorate" in November 1905. The Japanese Resident-General needed to rein in the Korean people's fervor for education-particularly the soaring number of private schools for national education and textbooks used at the schools. Korean intellectuals, who found that government schools and public schools were unsuitable for national education due to the Japanese Resident-General's supervision, established private schools all across Korea and wrote and compiled textbooks to be taught at the private schools. ${ }^{1}$

The Korean Empire, ruled by Japan as its protectorate from 1906 to 1910, was a de facto colony, as its diplomatic rights, and later domestic affairs, fell under the supervision of Japan. For efficient control of the Korean Empire, the Japanese Residency-General of Korea set out to control ideologies and information that could instill nationalistic or antiJapanese sentiments. This led to the implementation of a series of laws, such as the National Security Act and Newspaper Act in 1907, Regulations on Textbooks in 1908, and the Publication Act in 1909. Many studies have already revealed that the establishment of such a censorship system resulted in the suspension of publication of newspapers, magazines, and other print materials, as well as the confiscation and banning of textbooks and other publications. The contents of books that were banned for failing to meet the standards of inspection and censorship have also been researched as well. ${ }^{2}$

1 Kim Soyoung, 2010, “Taehan chegukki 'kukmin' hyŏngsŏngnon-gwa t'onghamnon yŏn'gu" (A study on the theory of the formation of "citizens" and the theory of integration in the Korean Empire), PhD diss., Korea University, 110-111.

2 Kang Yunho, 1973, Kaehwagi-ǔi kyogwayong tosŏ (Textbooks from the enlightenment period), Kyoyuk Ch'ulp'ansa; Yun Kŏnch'a, 1987, Han'guk kündae kyoyuk-üi sasang-gwa undong (Ideologies and movements of modern education in Korea), Ch'ŏngsa; Kim Bonghŭi, 1999, Han'guk kaehwagi sŏjŏk munhwa yŏn'gu (Research on the culture of books in Korea during the enlightenment period), Ihwa 
However, research on Japanese censorship has been concentrated on the colonial period even though print censorship and control of the media began with the rule of the Japanese Residency-General of Korea in 1906. This can be attributed to the fact that the censorship system became concrete and full-fledged in the colonial period, leaving plenty of documents and materials related to actual cases of censorship during this time.

In particular, most research papers focus on the censorship system and policies in relation to Japan's colonial policies after 1910. Japan implemented oppressive and restrictive policies to limit and control the freedom of the press and ideologies in Korea throughout the colonial period for efficient and strong rule over Korea. These control policies gave birth to the censorship systems not only in the print and publications sector, which includes newspapers, magazines, and textbooks, but also in other areas, such as film, art, and music. A close examination of research on control policies, censorship policies, and the censorship system, which muzzled the press and ideologies in colonial Korea, reveals that most studies focus on analyzing the Japanese Government-General's censorship laws and policies, censors, censorates, and the censorship process. Since the censorship system in colonial Korea was closely linked with Japan's censorship system, there are also comparative analysis studies that examined the impact Japan's censorship system had on its Korean counterpart, as well as the differences and similarities between the cen-

Yŏja Taehakkyo Ch'ulp'anbu; Yi Chungyŏn, 2001, “Ch'aek”-ŭi unmyŏngChosŏn-Ilje kangjömgi kŭmsŏ-ŭi sahoe sasangsa (The fate of "books" - the social and ideological history of banned books from Chosŏn Korea to the Japanese colonial period), Hyean; Chŏng Kŭnsik, 2003, "Singminjijŏk kŏmyŏl-ŭi yŏksajŏk kiwŏn: 1904-1910" (The historical origin of colonial censorship: 1904-1910), Sahoewa yŏksa 64; Ku Hŭichin, 2004 “Han'guk kŭndae kaehyŏkki-ŭi kyoyungnon-gwa kyoyuk kaep'yŏn" (Education theory and reorganization of education in the enlightenment period in Korea), PhD diss., Seoul National University; Yi Kyehyŏng, 2008, "Taehan chegukki t'onggambu-ŭi singmin kyoyuk ch^ongch'aek" (Research on the colonial education policies of the Japanese Residency-General of Korea in the Korean Empire), PhD diss., Kookmin University. 
sorship systems in Japan and Korea. ${ }^{3}$

Such diverse research on censorship in colonial Korea was possible thanks to the awareness that the censorship system, which controls ideologies, knowledge, and information, was an important and efficient policy for maintaining and strengthening Japan's colonial rule over Korea. Moreover, as the censorship system was active throughout the colonial period, plenty of sources and materials related to the censorship system are available today, which led to active research on the censorship system.

Among the studies on the censorship system in colonial Korea, of particular interest to this research in terms of topic and methodology are studies focusing on the analysis and examination of original publications that contain traces and notes from the censorship process. For instance, studies that have examined the censorship process through sample copies of magazines and novels published in colonial Korea as well as studies that have analyzed traces of censorship in newspapers and magazines

3 Chŏng Kŭnsik, 2005, "Iljeha kŏmyŏl kigu-wa kŏmyŏlgwan-ŭi pyŏndong" (Changes in the censorship agency and censors under the Japanese rule), Taedong munhwa yŏn'gu 51; Son Chiyŏn, 2007, “Singminji Chosŏn-esouŭi kŏmyŏl-ŭi sasang-gwa pangbŏp - kŏmyŏl charyojip kuch'uk kwajŏng-ŭl t'onghayŏ” (Concepts and methods of censorship in colonial Chosón through the structural process of compiling censorship documents), Han'guk munhak yŏn'gu 32; T'ongguk Taehakkyo Munhwa Haksurwŏn Han'guk Munhwa, 2010, Singminji sigi kŏmyŏlgwa Han'guk munhwa (Censorship and the Korean culture in colonial Korea), Tongguk Taehakkyo Ch'ulp'anbu; Kŏmyŏl Yŏn'guhoe, 2011, Singminji kŏmyŏl (Censoring Colonies), Somyŏng Ch’ulp'an; Chŏng Kŭnsik, 2013, "Singminji chŏnsi ch'ejeha-esŏŭi kŏmyŏl-gwa sŏnjŏn, kŭrigo tongwon" (Censorship, propaganda, and mobilization of colonial Korea in wartime), Sanghŏ hakbo 38; Chŏng Chinsŏk, 2014, Kŭkbi Chosŏn ch'ongdokbu-ŭi ŏllon kŏmyŏl-gwa t'anap -Ilbon-ŭi ch'imnyak-gwa yŏlgang seryǒk-ŭi ŏllon t'ongje (Confidential The Japanese Government-General of Korea and its censorship and oppression of the pressJapanese invasion and the press control of a powerful nation), Kŏmunikaeisyŏn buksŭ; Chŏng Kŭnsik et al., 2016, Kŏmyŏl-ŭi cheguk-munhwa-ŭi t'ongje-wa chaesaengsan (Empire of censorship - the control and reproduction of culture), P'urŭn Yŏksa. 
published in Taiwan, another Japanese colony, have various implications for this paper. In this study, I analyzed texts with traces of censorship and found the censors who had been in charge of inspecting the texts, leaving handwritten notes and censors' stamps, as well as the ways in which inspection and censorship were conducted through the censorship process and the post-censorship administrative process. Moreover, this study explored the censorship standards that the censors relied on to provide their recommendations regarding the revision and deletion of certain text. By analyzing not only the censorship standards and processes stipulated by law but also texts that have been censored, this paper paints a true portrait of the censorship system Japan imposed on colonial Korea. ${ }^{4}$

As mentioned previously, substantial research has been conducted on censorship in colonial Korea, as well as in the protectorate period, which this study covers, since the censorship system was instituted during this period. Just like the research on censorship in the colonial period, research on censorship during the protectorate period mainly concentrates on magazines and newspapers that have been discontinued due to censorship, publications that have been banned, the Japanese ResidencyGeneral's censorship policies and system, and the Korean people's protests and response to Japan's censorship policies. However, as mentioned earlier, documents and materials related to censorship from this period are relatively few in number compared to the documents from the colonial period. In particular, there were no print materials that had undergone inspection or censorship that were preserved until today, which resulted in a dearth of studies in this field.

Recently, however, copies of textbooks submitted to the Ministry of Education (Hakbu) for inspection in the protectorate period, which are a source of information regarding the state of censorship in the protectorate period, were newly discovered. Textbooks and documents that had been inspected by the Japanese Residency-General of Korea-inspection cop-

4 Kŏmyŏl yŏn'guhoe, 2011, Singminji kŏmyŏl: chedo, t'eksŭtŭ, silch'ŏn (Censorship in colonies: system, text, and performance), Somyŏng Ch'ulp'an. 
ies of textbooks from the Young Korean Academy (Heungsadan)-were included in the "Collection of new materials related to $\mathrm{Yu}$ Kilchun" that had been donated by Yu's descendants to the Korea University Museum in 2003. The "Collection of New materials related to Yu Kilchun" can be largely divided into "Books in Yu Kilchun's possession," "Yu Kilchun's documents," and "Yu Kilchun's letters." The inspection copies of textbooks were included in the "Books in Yu Kilchun's possession." $\mathrm{Yu}$ Kilchun was the founder of the Young Korean Academy, established in 1907 to provide modern education based on national education and elementary schooling and to foster talented individuals. Therefore it is highly likely for him to have been in possession of inspection copies of textbooks that had been written or compiled by the Young Korean Academy and people who had connections with the Young Korean Academy. ${ }^{5}$ This study is the first in a series of research that uses these newly discovered inspection copies of textbooks to examine actual cases of textbook inspection and censorship in Korea during the protectorate period and shed light on the historical significance of such activities. This paper will examine the following two main points:

First, this study will focus on the fact that Japan established and carried out censorship policies to control the press and publication during the protectorate period to strengthen its rule over Korea. Considering that the inspection and censorship system at this time was sustained into the colonial period, this study will analyze legislations about inspection and censorship to examine the early stages of the establishment of the censorship system in modern Korea.

Second, this study will provide a bibliographic analysis of the copies of textbooks that had been petitioned for inspection bibliographically. As mentioned earlier, inspection copies of textbooks from the protectorate period are new materials that had not been analyzed or used in research

5 Choe Tŏksu et al., 2015, Kŭndae Han'guk-ŭi kaehyŏk kusang-gwa Yu Kilchun (Reformation ideas in modern Korea and Yu Kilchun), Koryŏ Taehakkyo Ch'ulp'an Munhwawŏn, p 32-34. 
previously, and therefore a basic bibliographical analysis needs to be conducted for further research. General bibliographic information related to the textbooks, such as the author, publisher, year of publication, use, and table of contents, as well as parts that demonstrate the process of inspection need to be explored and explained. Moreover, it is also important to find out about the censors who had been in charge of textbook inspection as their names were recorded in the inspection copies, and investigate to see whether these textbooks have been published after inspection.

\section{Adoption of the Textbook Inspection and Censorship System}

With the issuance of the legislation for the organization of the Ministry of Education on March 25, 1895, and the establishment of the Editorial Bureau, the compilation of modern education textbooks began. However, specific regulations regarding textbook inspection had not yet been developed at the time. As an increasing number of privately compiled textbooks began to be published, the Japanese Residency-General of Korea stipulated in Article 4 of the Legislation on Middle School Education, issued in September 1900, that "for each subject in middle schools, textbooks that have been edited by the Ministry of Education or inspected by the Minister of Education" should be used. Article 12 of the same legislation also stipulated, "textbooks other than those that have been designated by the Ministry of Education shall not be used in government, public, and private middle schools." Japan, which had turned the Korean Empire into its protectorate through the Ǔlsa Treaty in November 1905, established the Residency-General in 1906 to rule Korea and began to exert its influence on the education of Koreans through the Ministry of Education. ${ }^{6}$

In 1907, Japan forced Emperor Kojong to abdicate the throne after the

6 Yi Kyehyŏng, 2008, “Taehan chegukki t'onggambu-ŭi singmin kyoyuk chŏngch'aek" (Research on the colonial education policies of the Japanese Residency-General of Korea in the Korean Empire). PhD diss., Kookmin University. 
Hague Secret Emissary Affair, signed the Third Japan-Korea Treaty, otherwise known in Korea as Chŏngmi Treaty, and disbanded the Korean military. Protests erupted in Seoul and all across Korea, and the Righteous Army, joined by the soldiers of the disbanded Korean military, rebelled and continued its struggle against Japan. The Korean press also persistently criticized Japan's policy toward Korea, condemning the Chŏngmi Treaty for violating the Korean Empire's autonomy. In response, the Japanese Resident-General used military force to suppress the Korean people's resistance and issued a series of legislations to limit and control the freedom of the press to regulate the Korean press that was vehemently criticizing the Japanese Resident-General while instilling loyalty and patriotism to the Korean Empire in Koreans. The Residency-General's control over the Korean press began with the Newspaper Act, issued on July 24, 1907..$^{7}$ The Newspaper Act signified the Korean people's loss of freedom of the press, as well as political freedom and the freedom of livelihood - it was an obstruction of Korea's rights and restraint over all the activities of its people. Following the Newspaper Act, the Japanese Residency-General of Korea enacted the National Security Act on July 27, $1907 .^{8}$

On August 26, 1908, the Japanese Residency-General issued the Decree Concerning Private Schools and specified a provision on textbooks in Article 6 of the decree. ${ }^{9}$ Soon afterwards, on September 1, 1908, the Regulations on Textbook Inspection were passed. ${ }^{10}$ The regulations had been enacted to control Korean education and textbooks, which were possibly the most effective and influential means to instill anti-Japanese sen-

7 Ku Han'guk kwanbo (Government gazette), July 27, Kwangmu 11, Legislation No.5 "Sinmunjibŏp" (Newspaper Act).

8 Choe Kiyŏng, 1981, “Kwangmu sinmunjibŏp-e kwanhan yŏn'gu” (A study on the Newspaper Act in the Kwangmu Era), Yǒksa hakbo 92, 42-29.

9 Ku Han'guk kwanbo, September 1, Yunghŭi 2, Decree No. 62 "Sarip hakkyoryŏng" (Decree Concerning Private Schools).

10 Ku Han'guk kwanbo, September 1, Yunghŭi 2, Decree of the Ministry of Education No. 16 “Kyogwayong tosŏ kŏmjŏng kyujŏng” (Regulations on Textbooks). 
timents and patriotism in young Korean students. The Ministry of Education began to deliberate on the contents of textbooks according to the Regulations on Textbook Inspection. The standards for textbook inspection were largely divided into three categories: political, social, and educational. In terms of political contents, textbooks were censored to remove "materials that hinder or criticize Korea-Japan relations and the amicable friendship between the two countries," "biased and artful materials that stir concern over the nation," and "materials that encourage the ideas of ostracizing Japan as well as records or statements that spur Koreans to foster animosity against the people of Japan or of other nations." Such standards for censorship suggest that the purpose of the Regulations on Textbook Inspection was to prevent the inclusion of any content that promoted or instilled anti-Japanese sentiments or patriotism in Koreans in textbooks.

In addition to the Regulations on Textbook Inspection, textbooks were also censored according to the Publication Act, the sixth legislation enacted by the Japanese Residency-General of Korea, issued on February 23, 1909. ${ }^{11}$ As a result, textbooks were subject to dual censorship of the Ministry of Education, which was in charge of textbook inspection, and the Ministry of Home Affairs (Naebu), which was responsible for the supervision of the Publication Act. ${ }^{12}$

The freedom of the press was strictly limited under such a censorship system. The censorship and inspection of published materials, in addition to the textbooks, resulted in the suspension and prohibition of the publication of Korean-owned newspapers and magazines, and further to confiscation of published materials. Led by intellectuals, Koreans criticized and protested against censorship laws issued by the Japanese ResidencyGeneral and their implementation. But there were others who preferred "compromise" and "conciliation." They undertook to write and publish

11 Sŏbukhakhoe Wŏlbo (Monthly Bulletin of Northwest Academic Association) 12, May 1909, "Kwanbojŏkyo (Summary of the official gazette).

12 Kim Soyoung, 2010, "Taehan chegukki," 113. 
books that met the inspection and censorship standards. Such instances of "compromises" and "conciliations" were also confirmed on the inspection copies of textbooks.

After issuing the Regulations on Textbook Inspection in 1908, the Japanese Residency-General of Korea began to inspect textbooks and identified problematic contents, most of which were statements that encouraged Koreans to foster anti-Japanese sentiments, patriotism, and the will to recover the national rights. Among the textbooks that were severely censored, "many contained materials or narrative methods that were not appropriate." One such example was ethics textbooks.

The Japanese Residency-General of Korea inspected ethics textbooks and criticized them for containing a number of theories that were difficult for students to understand, such as national laws and duties, when the textbooks should be focused on easier materials that students encounter in their daily lives as they were meant to educate young students, mainly in elementary schools. The Residency-General further asserted that contents that need to be addressed in ethics textbooks were Confucian virtues, such as filial piety, loyalty, decorum, and honor, as well as virtues required of modern people, such as diligence and cleanliness. National laws and duties should be taught to students as responsibilities of citizens once they have gained further knowledge. ${ }^{13}$

Any political materials in textbooks were also considered problematic. The Residency-General saw political contents in textbooks as proof that Koreans do not understand that politics and education should be separate, and that Koreans wish to use such textbooks for other purposes. Such a political nature was considered to be more prominent particularly in textbooks, for subjects such as ethics, Korean language, classical Chinese (hanmun), and history, and the Residency-General listed the problematic contents as follows:

13 "Kyokwasŏ-ŭi naeyong-e kwanhan chosa" (Investigation related to the contents of textbooks), Kiho Hŭnghakhoe Wŏlbo (Monthly Bulletin of Capital and Central Region Association for Promotion of Learning) Vol. 12, July 1909, 39. 
One, contents that outline the current situation of the Korean Empire; two, vehement assertions for independence and recovery of national sovereignty; three, warnings over the future of the Korean Empire using examples of other nations; four, satirical expressions that it is impossible not to request for help from other nations; five, statements that arouse hostility against Japan and other nations by exaggerating historical events concerning Japan and other nations; six, contents that disturb the diplomatic relations between Korea and Japan by narrating recent Korean history (in words of resentment); seven, contents advocating anti-foreignism by asserting that maintaining one's own national language, customs, and practices while imitating other nations is impossible; eight, seditious speech based on the theory of the state and deontology; and lastly, contents that encourage patriotism. ${ }^{14}$

The inspection standards regarding contents about patriotism were particularly noteworthy. The Ministry of Education criticized the contents concerning patriotism in textbooks that did not meet their standards, saying the descriptions of patriotism in textbooks were not about "true patriotism," and condemned the textbooks for delivering incorrect information on expressing patriotism. ${ }^{15}$ The "true patriotism" that the Ministry of Education advocated was not political awareness such as liberty and independence or nationalism, but virtues such as diligence to achieve national prosperity and military prowess by fulfilling one's duties for the wealth and power of the nation.

As such, the Japanese Residency-General of Korea established and enacted laws and standards for textbook inspection and censorship to prevent education among the Korean people that encouraged anti-Japanese sentiments and aroused national consciousness. This meant that Japan needed to implement policies to stop Korean resistance or criticism

14 Ibid., 40-42.

15 Ibid., $42-43$. 
against Japanese rule, so that it could achieve its ultimate goal of annexing the Korean Empire, and one of these policies was the establishment of the inspection and censorship system for controlling education and the press.

\section{Review of Actual Cases of Textbook Inspection Using Copies of Textbooks Submitted for Inspection}

As mentioned in the previous sections, most studies that have explored the Japanese Residency-General's textbook inspection system mainly analyzed the inspection standards stipulated in the Regulations for Textbook Inspection. These studies also examined the list of textbooks that were confiscated or banned from publication and their contents to indirectly identify the types of materials the Residency-General attempted to wipe from the textbooks. However, the lack of physical examples, such as copies of textbooks submitted for inspection, had resulted in a status quo for research on textbook inspection and censorship.

Fortunately, the discovery of the copies of textbooks that were submitted to the Ministry of Education for inspection has led to a breakthrough in the research on textbook inspection and censorship in Korea during the protectorate period. The following table contains a list of textbooks that had been submitted to the Ministry of Education for inspection.

Table 1. List of Copies of Textbooks Submitted for Inspection

\begin{tabular}{l|l|l|l|l|l|l}
\hline Textbook & $\begin{array}{c}\text { No of } \\
\text { Vol- } \\
\text { umes }\end{array}$ & $\begin{array}{c}\text { Year of } \\
\text { Inspec- } \\
\text { tion }\end{array}$ & $\begin{array}{c}\text { Lan- } \\
\text { guage }\end{array}$ & $\begin{array}{c}\text { Author (Pub- } \\
\text { lisher) }\end{array}$ & $\begin{array}{c}\text { Inspec- } \\
\text { tor }\end{array}$ & Notes \\
\hline $\begin{array}{l}\text { Ch'odŭng } \\
\text { nongóp } \\
\text { taeyo } 1\end{array}$ \\
$\begin{array}{l}\text { (Elemen- } \\
\text { tary Agri- } \\
\text { cuture1) }\end{array}$
\end{tabular}




\begin{tabular}{|c|c|c|c|c|c|c|}
\hline & & & & & & $\begin{array}{l}\text { der the "name of } \\
\text { the censor" but } \\
\text { stamped with "Oda } \\
\text { (小田) on notes for } \\
\text { revision or deletion } \\
\text { of text }\end{array}$ \\
\hline $\begin{array}{l}\text { Ch'odŭng } \\
\text { nongŏp } \\
\text { taeyo } 2 \\
\text { (Elemen- } \\
\text { tary agri- } \\
\text { culture 2) }\end{array}$ & 1 & 1909 & $\begin{array}{l}\text { Mixed } \\
\text { script }\end{array}$ & $\begin{array}{l}\text { Pak Chŏng- } \\
\text { tong (Hong } \\
\text { Kichu) }\end{array}$ & $\begin{array}{l}\text { Fuku- } \\
\text { shima } \\
\text { (福島) }\end{array}$ & $\begin{array}{l}\text { Petitioned for in- } \\
\text { spection, for fourth } \\
\text { grade private } \\
\text { school students, } \\
\text { correc- } \\
\text { tions,"Fukushima } \\
\text { (福島)" recorded } \\
\text { under the "name of } \\
\text { the censor" but } \\
\text { stamped with “Oda } \\
\text { (小田) onnotes for } \\
\text { revision or deletion } \\
\text { of text }\end{array}$ \\
\hline $\begin{array}{l}\text { Pot'ongha } \\
k \text { susinsŏ } 1 \\
\text { (Ordinary } \\
\text { (elemen- } \\
\text { tary) eth- } \\
\text { ics } 1 \text { ) }\end{array}$ & 1 & $\begin{array}{l}\text { Un- } \\
\text { known }\end{array}$ & $\begin{array}{l}\text { Mixed } \\
\text { script }\end{array}$ & $\begin{array}{l}\text { Presumed to } \\
\text { be Pak } \\
\text { Chŏngtong or } \\
\text { Yu Kilchun }\end{array}$ & & $\begin{array}{l}\text { Petitioned for in- } \\
\text { spection, for ordi- } \\
\text { nary school stu- } \\
\text { dents in first grade, } \\
\text { corrections, strips } \\
\text { of notes, revisions }\end{array}$ \\
\hline $\begin{array}{l}\text { Pot'ongha } \\
\text { k susinsŏ } 2 \\
\text { (Ordinary } \\
\text { ethics 2) }\end{array}$ & 1 & $\begin{array}{l}\text { Un- } \\
\text { known }\end{array}$ & $\begin{array}{l}\text { Mixed } \\
\text { script }\end{array}$ & $\begin{array}{l}\text { Presumed to } \\
\text { be Pak } \\
\text { Chŏngtong or } \\
\text { Yu Kilchun }\end{array}$ & & $\begin{array}{l}\text { Petitioned for in- } \\
\text { spection, for se- } \\
\text { cond grade ordi- } \\
\text { nary school stu- } \\
\text { dents, corrections, } \\
\text { strips of notes, } \\
\text { revisions }\end{array}$ \\
\hline $\begin{array}{l}\text { Pot'ongha } \\
\text { k susinsŏ } 3 \\
\text { (Ordinary } \\
\text { ethics 3) }\end{array}$ & 1 & $\begin{array}{l}\text { Un- } \\
\text { known }\end{array}$ & $\begin{array}{l}\text { Mixed } \\
\text { script }\end{array}$ & $\begin{array}{l}\text { Presumed to } \\
\text { be Pak } \\
\text { Chŏngtong or } \\
\text { Yu Kilchun }\end{array}$ & & $\begin{array}{l}\text { Petitioned for in- } \\
\text { spection, for third } \\
\text { grade ordinary } \\
\text { school students, } \\
\text { corrections, strips } \\
\text { of notes, revisions }\end{array}$ \\
\hline $\begin{array}{l}\text { Pot'ongha } \\
\text { k susinsŏ } 4 \\
\text { (Ordinary } \\
\text { ethics } 4 \text { ) }\end{array}$ & 1 & $\begin{array}{l}\text { Un- } \\
\text { known }\end{array}$ & $\begin{array}{l}\text { Mixed } \\
\text { script }\end{array}$ & $\begin{array}{l}\text { Presumed to } \\
\text { be Pak } \\
\text { Chŏngtong or } \\
\text { Yu Kilchun }\end{array}$ & & $\begin{array}{l}\text { Petitioned for in- } \\
\text { spection, for fourth } \\
\text { grade ordinary } \\
\text { school students, }\end{array}$ \\
\hline
\end{tabular}




\begin{tabular}{|c|c|c|c|c|c|}
\hline & & & & & $\begin{array}{l}\text { corrections, strips } \\
\text { of notes, revisions }\end{array}$ \\
\hline $\begin{array}{l}\text { Ch'odŭng } \\
\text { (hak) } \\
\text { pon'guk } \\
\text { yak (yok) } \\
\text { sa 1 } \\
\text { (Elemen- } \\
\text { tary sur- } \\
\text { vey of } \\
\text { Korean } \\
\text { history 1) }\end{array}$ & 1 & 1908 & $\begin{array}{l}\text { Mixed } \\
\text { script }\end{array}$ & $\begin{array}{l}\text { Presumed to } \\
\text { be Young } \\
\text { Korean Acad- } \\
\text { emy }\end{array}$ & $\begin{array}{l}\text { Petitioned for in- } \\
\text { spection, approved } \\
\text { for publication, } \\
\text { corrections }\end{array}$ \\
\hline $\begin{array}{l}\text { Chodŭng } \\
\text { (hak) } \\
\text { pon'guk } \\
\text { yak (yók) } \\
\text { sa } 2 \text { (Ele- } \\
\text { mentary } \\
\text { survey of } \\
\text { Korean } \\
\text { history 2) }\end{array}$ & 1 & 1908 & $\begin{array}{l}\text { Mixed } \\
\text { script }\end{array}$ & $\begin{array}{l}\text { Presumed to } \\
\text { be Young } \\
\text { Korean Acad- } \\
\text { emy }\end{array}$ & $\begin{array}{l}\text { Petitioned for in- } \\
\text { spection, approved } \\
\text { for publication, for } \\
\text { fourth grade pri- } \\
\text { vate school stu- } \\
\text { dents, corrections }\end{array}$ \\
\hline $\begin{array}{l}\text { Ch'odŭng } \\
\text { (hak) } \\
\text { pon'guk } \\
\text { yak (yŏk) } \\
\text { sa } 3 \text { (Ele- } \\
\text { mentary } \\
\text { survey of } \\
\text { Korean } \\
\text { history 3) }\end{array}$ & 1 & 1908 & $\begin{array}{l}\text { Mixed } \\
\text { script }\end{array}$ & \begin{tabular}{|l|} 
Presumed to \\
be Young \\
Korean Acad- \\
emy
\end{tabular} & Corrections \\
\hline $\begin{array}{l}\text { Ch'odüng } \\
\text { pon'guk } \\
\text { chiri } 1 \\
\text { (Elemen- } \\
\text { tary Kore- } \\
\text { an geogra- } \\
\text { phy 1) }\end{array}$ & 1 & 1909 & $\begin{array}{l}\text { Mixed } \\
\text { script }\end{array}$ & $\begin{array}{l}\text { Young Kore- } \\
\text { an Academy } \\
\text { Editorial De- } \\
\text { partment } \\
\text { (Tong- } \\
\text { mun'gwan) }\end{array}$ & $\begin{array}{l}\text { Corrections, strips } \\
\text { of notes }\end{array}$ \\
\hline $\begin{array}{l}\text { Ch'odüng } \\
\text { pon'guk } \\
\text { chiri 1 } \\
\text { (Elemen- } \\
\text { tary Kore- }\end{array}$ & 1 & 1909 & $\begin{array}{l}\text { Mixed } \\
\text { script }\end{array}$ & $\begin{array}{l}\text { Young Kore- } \\
\text { an Academy } \\
\text { Editorial De- } \\
\text { partment } \\
\text { (Tong- }\end{array}$ & $\begin{array}{l}\text { Approved for pub- } \\
\text { lication, correc- } \\
\text { tions, strips of } \\
\text { notes, censor's } \\
\text { stamp }\end{array}$ \\
\hline
\end{tabular}




\begin{tabular}{|c|c|c|c|c|c|}
\hline $\begin{array}{l}\text { an geogra- } \\
\text { phy 1) }\end{array}$ & & & & mun'gwan) & \\
\hline $\begin{array}{l}\text { Ch'odŭng } \\
\text { pon'guk } \\
\text { chiri } 2 \\
\text { (Elemen- } \\
\text { tary Kore- } \\
\text { an geogra- } \\
\text { phy 2) }\end{array}$ & 1 & 1909 & $\begin{array}{l}\text { Mixed } \\
\text { script }\end{array}$ & $\begin{array}{l}\text { Young Kore- } \\
\text { an Academy } \\
\text { Editorial De- } \\
\text { partment } \\
\text { (Tong- } \\
\text { mun'gwan) }\end{array}$ & $\begin{array}{l}\text { Petitioned for in- } \\
\text { spection, approved } \\
\text { for publication, } \\
\text { corrections, cen- } \\
\text { sor's stamp }\end{array}$ \\
\hline $\begin{array}{l}\text { Ch'odŭng } \\
\text { igwa } 1 \\
\text { (Elemen- } \\
\text { tary sci- } \\
\text { ence 1) }\end{array}$ & 1 & 1910 & $\begin{array}{l}\text { Mixed } \\
\text { script }\end{array}$ & $\begin{array}{l}\text { Pak Chŏng- } \\
\text { tong, chief } \\
\text { editor of the } \\
\text { Young Kore- } \\
\text { an Academy } \\
\text { (Hong Kichu, } \\
\text { Tong- } \\
\text { mun'gwan) }\end{array}$ & $\begin{array}{l}\text { For third grade } \\
\text { private school } \\
\text { students, petitioned } \\
\text { for inspection, } \\
\text { approved by the } \\
\text { Ministry of Educa- } \\
\text { tion, corrections, } \\
\text { censor's stamp, } \\
\text { includes the peti- } \\
\text { tion form }\end{array}$ \\
\hline $\begin{array}{l}\text { Ch'odŭng } \\
\text { igwa } 2 \\
\text { (Elemen- } \\
\text { tary sci- } \\
\text { ence 2) }\end{array}$ & 1 & 1910 & $\begin{array}{l}\text { Mixed } \\
\text { script }\end{array}$ & $\begin{array}{l}\text { Pak Chŏng- } \\
\text { tong, chief } \\
\text { editor of the } \\
\text { Young Kore- } \\
\text { an Academy } \\
\text { (Hong Kichu, } \\
\text { Tong- } \\
\text { mun'gwan) }\end{array}$ & $\begin{array}{l}\text { For third grade and } \\
\text { fourth grade pri- } \\
\text { vate school stu- } \\
\text { dents, petitioned } \\
\text { for inspection, } \\
\text { approved by the } \\
\text { Ministry of Educa- } \\
\text { tion, corrections, } \\
\text { censor's stamp }\end{array}$ \\
\hline $\begin{array}{l}\text { Ch'odŭng } \\
\text { igwa } 3 \\
\text { (Elemen- } \\
\text { tary sci- } \\
\text { ence 3) }\end{array}$ & 1 & 1910 & $\begin{array}{l}\text { Mixed } \\
\text { script }\end{array}$ & $\begin{array}{l}\text { Pak Chŏng- } \\
\text { tong, chief } \\
\text { editor of the } \\
\text { Young Kore- } \\
\text { an Academy } \\
\text { (Hong Kichu, } \\
\text { Tong- } \\
\text { mun'gwan) }\end{array}$ & $\begin{array}{l}\text { For fourth grade } \\
\text { private school } \\
\text { students, petitioned } \\
\text { for inspection, } \\
\text { approved by the } \\
\text { Ministry of Educa- } \\
\text { tion, corrections }\end{array}$ \\
\hline $\begin{array}{l}\text { Ch'odŭng } \\
\text { sohak } \\
\text { tokbon } 1 \\
\text { (Elemen- } \\
\text { tary read- } \\
\text { ing text- }\end{array}$ & 1 & & $\begin{array}{l}\text { Han'gŭ } \\
1\end{array}$ & Unknown & $\begin{array}{l}\text { Presumed to be a } \\
\text { draft of the text- } \\
\text { book }\end{array}$ \\
\hline
\end{tabular}




\begin{tabular}{|c|c|c|c|c|c|c|}
\hline book 1) & & & & & & \\
\hline $\begin{array}{l}\text { Yuch'i } \\
\text { tokbon } 1 \\
\text { (Reading } \\
\text { for chil- } \\
\text { dren 1) }\end{array}$ & 2 & 1909 & $\begin{array}{l}\text { Han'gŭ } \\
1\end{array}$ & $\begin{array}{l}\text { Pak Chŏng- } \\
\text { tong, chief } \\
\text { editor of the } \\
\text { Young Kore- } \\
\text { an Academy } \\
\text { (Hong Kichu, } \\
\text { Tong- } \\
\text { mun'gwan) }\end{array}$ & $\begin{array}{l}\text { Oda (小 } \\
\text { 田, main } \\
\text { inspec- } \\
\text { tor), } \\
\text { Uemura } \\
\text { (上村), } \\
\text { Hyŏn } \\
\text { Hŏn }\end{array}$ & $\begin{array}{l}\text { Petitioned for in- } \\
\text { spection, correc- } \\
\text { tions, strips of } \\
\text { notes, censor's } \\
\text { stamp }\end{array}$ \\
\hline $\begin{array}{l}\text { Yuch'i } \\
\text { tokbon } 2 \\
\text { (Reading } \\
\text { for chil- } \\
\text { dren 2) }\end{array}$ & 1 & 1909 & $\begin{array}{l}\text { Han'gŭ } \\
1\end{array}$ & $\begin{array}{l}\text { Pak Chŏng- } \\
\text { tong, chief } \\
\text { editor of the } \\
\text { Young Kore- } \\
\text { an Academy } \\
\text { (Hong Kichu, } \\
\text { Tong- } \\
\text { mun'gwan) }\end{array}$ & $\begin{array}{l}\text { Oda (小 } \\
\text { 田, main } \\
\text { inspec- } \\
\text { tor), } \\
\text { Uemura } \\
\text { (上村), } \\
\text { Hyŏn } \\
\text { Hŏn }\end{array}$ & $\begin{array}{l}\text { Petitioned for in- } \\
\text { spection, correc- } \\
\text { tions, strips of } \\
\text { notes, censor's } \\
\text { stamp }\end{array}$ \\
\hline $\begin{array}{l}\text { Yuch'i } \\
\text { tokbon } 3 \\
\text { (Reading } \\
\text { for chil- } \\
\text { dren 3) }\end{array}$ & 1 & 1909 & $\begin{array}{l}\text { Han'gŭ } \\
1\end{array}$ & $\begin{array}{l}\text { Pak Chŏng- } \\
\text { tong, chief } \\
\text { editor of the } \\
\text { Young Kore- } \\
\text { an Academy } \\
\text { (Hong Kichu, } \\
\text { Tong- } \\
\text { mun'gwan) }\end{array}$ & $\begin{array}{l}\text { Oda (小 } \\
\text { 田, main } \\
\text { inspec- } \\
\text { tor), } \\
\text { Uemura } \\
\text { (上村), } \\
\text { Hyŏn } \\
\text { Hŏn }\end{array}$ & $\begin{array}{l}\text { Petitioned for in- } \\
\text { spection, correc- } \\
\text { tions, strips of } \\
\text { notes, censor's } \\
\text { stamp }\end{array}$ \\
\hline $\begin{array}{l}\text { Yuch'i } \\
\text { tokbon } 1 \\
\text { (Reading } \\
\text { for chil- } \\
\text { dren 1) }\end{array}$ & 1 & 1909 & Han'gŭ & $\begin{array}{l}\text { Pak Chŏng- } \\
\text { tong, chief } \\
\text { editor of the } \\
\text { Young Kore- } \\
\text { an Academy } \\
\text { (Hong Kichu, } \\
\text { Tong- } \\
\text { mun'gwan) }\end{array}$ & $\begin{array}{l}\text { Oda (小 } \\
\text { 田, main } \\
\text { inspec- } \\
\text { tor), } \\
\text { Uemura } \\
\text { (上村), } \\
\text { Hyŏn } \\
\text { Hŏn }\end{array}$ & $\begin{array}{l}\text { Presumed to have } \\
\text { been revised after } \\
\text { petitioning for } \\
\text { inspection, correc- } \\
\text { tions, strips of } \\
\text { notes }\end{array}$ \\
\hline $\begin{array}{l}\text { Yuch'i } \\
\text { tokbon } 3 \\
\text { (Reading } \\
\text { for chil- } \\
\text { dren 3) }\end{array}$ & 1 & 1909 & $\begin{array}{l}\text { Han'gŭ } \\
1\end{array}$ & $\begin{array}{l}\text { Pak Chŏng- } \\
\text { tong, chief } \\
\text { editor of the } \\
\text { Young Kore- } \\
\text { an Academy } \\
\text { (Hong Kichu, } \\
\text { Tong- } \\
\text { mun'gwan) }\end{array}$ & $\begin{array}{l}\text { Oda (小 } \\
\text { 田, main } \\
\text { inspec- } \\
\text { tor), } \\
\text { Uemura } \\
\text { (上村), } \\
\text { Hyŏn } \\
\text { Hŏn }\end{array}$ & $\begin{array}{l}\text { Presumed to have } \\
\text { been revised after } \\
\text { petitioning for } \\
\text { inspection, correc- } \\
\text { tions, strips of } \\
\text { notes, illustrations }\end{array}$ \\
\hline
\end{tabular}




\begin{tabular}{|c|c|c|c|c|c|c|}
\hline $\begin{array}{l}\text { Ch'odüng } \\
\text { hak tokbon } \\
1,2,3,4 \\
\text { (Elemen- } \\
\text { tary read- } \\
\text { ing } 1,2,3 \text {, } \\
\text { 4) }\end{array}$ & 1 & 1909 & $\begin{array}{l}\text { Mixed } \\
\text { script }\end{array}$ & $\begin{array}{l}\text { Pak Chŏng- } \\
\text { tong, chief } \\
\text { editor of the } \\
\text { Young Kore- } \\
\text { an Academy } \\
\text { (Hong Kichu, } \\
\text { Tong- } \\
\text { mun'gwan) }\end{array}$ & $\begin{array}{l}\text { Ueda } \\
\text { (上田, } \\
\text { main } \\
\text { inspec- } \\
\text { tor), } \\
\text { Uemura } \\
\text { (上村), } \\
\text { Yi } \\
\text { Chongha } \\
\text { (deleted) }\end{array}$ & $\begin{array}{l}\text { Bound volumes of } \\
\text { Elementary Read- } \\
\text { ing 1, 2, 3, and 4, } \\
\text { petitioned for in- } \\
\text { spection }\end{array}$ \\
\hline $\begin{array}{l}\text { Ch'odüng } \\
\text { hak tokbon } \\
1 \text { (Ele- } \\
\text { mentary } \\
\text { Reading 1) }\end{array}$ & 1 & 1909 & $\begin{array}{l}\text { Mixed } \\
\text { script }\end{array}$ & $\begin{array}{l}\text { Pak Chŏng- } \\
\text { tong, chief } \\
\text { editor of the } \\
\text { Young Kore- } \\
\text { an Academy } \\
\text { (Hong Kichu, } \\
\text { Tong- } \\
\text { mun'gwan) }\end{array}$ & $\begin{array}{l}\text { Ueda } \\
\text { (上田, } \\
\text { main } \\
\text { inspec- } \\
\text { tor), } \\
\text { Uemura } \\
\text { (上村) }\end{array}$ & $\begin{array}{l}\text { Petitioned for in- } \\
\text { spection, for first } \\
\text { grade elementary } \\
\text { school students, } \\
\text { corrections, strips } \\
\text { of notes, censor, } \\
\text { censor's stamp }\end{array}$ \\
\hline $\begin{array}{l}\text { Ch'odŭng } \\
\text { hak tokbon } \\
2 \text { (Ele- } \\
\text { mentary } \\
\text { reading } 2)\end{array}$ & 1 & 1909 & $\begin{array}{l}\text { Mixed } \\
\text { script }\end{array}$ & $\begin{array}{l}\text { Pak Chŏng- } \\
\text { tong, chief } \\
\text { editor of the } \\
\text { Young Kore- } \\
\text { an Academy } \\
\text { (Hong Kichu, } \\
\text { Tong- } \\
\text { mun'gwan) }\end{array}$ & $\begin{array}{l}\text { Ueda } \\
\text { (上田, } \\
\text { main } \\
\text { inspec- } \\
\text { tor), } \\
\text { Uemura } \\
\text { (上村) }\end{array}$ & $\begin{array}{l}\text { Petitioned for in- } \\
\text { spection, for se- } \\
\text { cond grade ele- } \\
\text { mentary school } \\
\text { students, correc- } \\
\text { tions, strips of } \\
\text { notes, censor's } \\
\text { stamp }\end{array}$ \\
\hline $\begin{array}{l}\text { Ch'odüng } \\
\text { hak tokbon } \\
3 \text { (Ele- } \\
\text { mentary } \\
\text { reading 3) }\end{array}$ & 1 & 1909 & $\begin{array}{l}\text { Mixed } \\
\text { script }\end{array}$ & $\begin{array}{l}\text { Pak Chŏng- } \\
\text { tong, chief } \\
\text { editor of the } \\
\text { Young Kore- } \\
\text { an Academy } \\
\text { (Hong Kichu, } \\
\text { Tong- } \\
\text { mun'gwan) }\end{array}$ & $\begin{array}{l}\text { Ueda } \\
\text { (上田, } \\
\text { main } \\
\text { inspec- } \\
\text { tor), } \\
\text { Uemura } \\
\text { (上村) }\end{array}$ & $\begin{array}{l}\text { Petitioned for in- } \\
\text { spection, for third } \\
\text { grade elementary } \\
\text { school students, } \\
\text { corrections, strips } \\
\text { of notes, censor's } \\
\text { stamp }\end{array}$ \\
\hline $\begin{array}{l}\text { Ch'odüng } \\
\text { hak tokbon } \\
4 \text { (Ele- } \\
\text { mentary } \\
\text { reading 4) }\end{array}$ & 1 & 1909 & $\begin{array}{l}\text { Mixed } \\
\text { script }\end{array}$ & $\begin{array}{l}\text { Pak Chŏng- } \\
\text { tong, chief } \\
\text { editor of the } \\
\text { Young Kore- } \\
\text { an Academy } \\
\text { (Hong Kichu, } \\
\text { Tong- } \\
\text { mun'gwan) }\end{array}$ & $\begin{array}{l}\text { Ueda } \\
\text { (上田, } \\
\text { main } \\
\text { inspec- } \\
\text { tor), } \\
\text { Uemura } \\
\text { (上村) }\end{array}$ & $\begin{array}{l}\text { Petitioned for in- } \\
\text { spection, for fourth } \\
\text { grade elementary } \\
\text { school students, } \\
\text { corrections, strips } \\
\text { of notes, censor's } \\
\text { stamp }\end{array}$ \\
\hline
\end{tabular}


A close look at Table 1 reveals that most of the copies of textbooks that were submitted for inspection had been written by Pak Chŏngtong and published by the Tongmun'gwan. Both Pak Chŏngtong and Tongmun'gwan were related to the Young Korean Academy. Pak Chŏngtong was the chief editor of the Young Korean Academy, ${ }^{16}$ and Tongmun'gwan was a printer established in affiliation with the Young Korean Academy, which became a full-fledged publisher in later years. ${ }^{17}$ The Young Korean Academy was founded by Yu Kilchun in November 1907 for the purpose of promoting national education to turn all Koreans into sŏnbi (virtuous scholars). ${ }^{18}$ In the "Rules of the Young Korean Academy," the main tasks of the institution for national education were listed as follows:

Article 2. This organization is dedicated to researching ways to bring education to ordinary citizens.

Article 3. This organization engages in the following activities (written on the left-hand side) to realize the purpose stated in the previous article:

a. compiling and publishing ordinary school textbooks for Koreans,

b. fostering people who can serve as teachers,

c. finding ways to maintain and sustain existing schools, as well as researching and accomplishing the approach to establish and maintain schools in regions without established schools,

16 Pak Chŏngtong graduated from Hansŏng Normal School. He taught at government-owned elementary schools, public elementary schools in Namwŏn-gun, and Hansŏng Normal School, and also lectured on science at Hŭimun Ǔisuk. He was active in various organizations including Kiho Hŭnghakhoe, Kyonam Hakhoe (Southeast Academic Association), and the Young Korean Academy.

17 This Tongmun'gwan is different from the Tongmun'gwan Foreign Language School.

18 "Hŭngsadan ch'uijisŏ mit Hŭngsadan kyuch'ik-ŭn," (Prospectus and rules of the Young Korean Academy), Yu Kilchun chŏnsŏ 2 (Collected Works of Yu Kilchun) (Yu Kilchun chŏnsŏ p'yŏnch'an wiwŏnhoep'yŏn, Iljogak, 1971). 
d. accepting requests and providing as much help as possible for the hiring of teachers and purchase of textbooks for each school,

e. gradually establishing a mandatory education system for ordinary citizens according to the educational purpose of this organization.

As stipulated in the rules, the foremost purpose of the founding of the Young Korean Academy was to implement national education to improve the character of Korean citizens. Writing and compiling textbooks necessary for national education were another goal to achieve for the Young Korean Academy.

An editorial published in Hwangsŏng sinmun (Capital Gazette) on May 3, 1908, titled "Necessary Projects for the Young Korean Academy" also mentioned the compilation of textbooks as an urgent task for the Young Korean Academy:

Textbooks are necessities for scholars; same as fields are necessities for farmers and machines for factory workers. If there are no decent textbooks, how can refined learning exist? I observed the education systems of civilized nations around the world and found that decent textbooks met the demand for ordinary middle school, high school, as well as vocational schools thanks to the painstaking labor and careful studies of competent scholars who were knowledgeable in each subject. In Korea, education had only been centered on one subject-Chinese - in the past.

Textbooks in elementary schools only consisted of Chŏnjamun (The Thousand Character Classic), Tongmong sŏnsŭp (The Primer for Youth), Saryak (Book of Brief History), and T'onggam (Comprehensive Book in Aid of Governance). In the recent years, several educators have published textbooks for various subjects, but these are by no means sufficient for elementary education. Therefore how can compilation and publication of textbooks not be a pressing pro- 
ject for an educational institution today? (The rest is omitted) ${ }^{19}$

It is clear that $\mathrm{Yu}$ Kilchun played a pivotal role in leading the compilation of textbooks. An article from the Hwangsŏng sinmun on December 28, 1907, noted that Yu was in charge of publishing textbooks, an important project of the Young Korean Academy: "The purpose of the founding of the Young Korean Academy has been stated in the previous issue. Recently, the academy established an office, and Yu Kilchun, the director of the Academy, has been working and even sleeping in the office to edit textbooks for elementary schools. He is in charge of compiling Korean reading textbooks and ethics textbooks, and he also directs and helps with editing as well." 20

The list of textbooks that had been authored and published by $\mathrm{Yu}$ Kilchun and other people with connection to the Young Korean Academy is as follows:

The comparison of the list of textbooks in Table 2 and the list of inspection copies of textbooks shows that while some textbooks passed the inspection and were published, others were not approved for publication. For instance, a copy of Yuch'i tokbon (Children's Reader) was submitted to the Ministry of Education for inspection, but it did not pass the censors and therefore was not published. Among the textbooks from the enlightenment period that have been preserved until today, there are none that are titled Yuch'i tokbon. Since it was possible that the title of the textbook was changed after inspection, I examined the contents of published elementary reading books alongside the inspection copy. However, none of the books matched the inspection copy. Also, an article in the Hwangsŏng sinmun around this time seemed to contain information regarding the inspection of Yuch'i tokbon, implying the possibility that Yuch'i tokbon did not pass the inspection.

19 “Editorial," Hwangsŏng sinmun, May 3, 1908.

20 “Miscellaneous news," Hwangsŏng sinmun, December 28, 1907. 
Table 2. List of Textbooks Written and Published by the Young Korean Academy and Related People

\begin{tabular}{|c|c|c|}
\hline Author & Textbook (Year of Publication) & $\begin{array}{c}\text { Inspection Copy } \\
\text { (Year of Inspection) }\end{array}$ \\
\hline $\begin{array}{l}\text { Young Kore- } \\
\text { an Academy }\end{array}$ & $\begin{array}{l}\text { Ch'odüng pon'guk chiri, (Elementary Ko- } \\
\text { rean Geography, 1909), Ch'odüng pon'guk } \\
\text { yaksa (1909, Elementary Korean history), } \\
\text { Sinch'anso pangmurhak (1908, New text- } \\
\text { book of natural history for beginners) }\end{array}$ & $\begin{array}{l}\text { Ch'odüng pon'guk } \\
\text { chiri (Elementary Ko- } \\
\text { rean geography) in- } \\
\text { spection copy (1909, } \\
\text { approved, authorized } \\
\text { for publication), } \\
\text { Ch'odŭng pon'guk yak } \\
\text { (yok) sa (Elementary } \\
\text { survey of Korean histo- } \\
\text { ry) inspection copy } \\
\text { (approved, authorized } \\
\text { for publication) }\end{array}$ \\
\hline Yu Kilchun & $\begin{array}{l}\text { Huryetuik taewang ch'illyŏnchŏnsa (1908, } \\
\text { History of the Seven Years' War under } \\
\text { Frederick the Great), Taehan munjŏn } \\
\text { (1908, Korean grammar), Nodong yahak } \\
\text { tokbon (1908, Laborer's night school read- } \\
\text { ing book), Yŏngbŏp rot'o karimiya chŏnsa } \\
\text { (1908, History of England, France, Russia, } \\
\text { Turkey, and Crimea). }\end{array}$ & $\begin{array}{l}\text { Pot'onghak susinsŏ } \\
\text { (presumed to have } \\
\text { been written by Yu } \\
\text { Kilchun or Pak Chŏng- } \\
\text { tong) }\end{array}$ \\
\hline $\begin{array}{l}\text { Pak Chŏng- } \\
\text { tong }\end{array}$ & $\begin{array}{l}\text { Ch'odŭng taedong yŏksa (1909, Elemen- } \\
\text { tary Korean history), Ch'odüng susin } \\
\text { (1909, Elementary ethics), Ch'odüng } \\
\text { pon'guk chiri (1909, Elementary Korean } \\
\text { geography), Ch'odŭng ihwahak (1908, } \\
\text { Elementary physics and chemis- } \\
\text { try),Sinch'an ihwahak (1908, New text- } \\
\text { book for physics and chemistry), } \\
\text { Sinch'anch'ǒkdokwanp'yon (1909, New } \\
\text { textbook on letter writing), Sinch'an } \\
\text { kajŏnghak (1907, New textbook for home } \\
\text { economics), Ch'an ihwahak (1908, Phys- } \\
\text { ics and chemistry), Ch'odüng pon'guk } \\
\text { yaksa (1909, Elementary survey of Korean } \\
\text { history) }\end{array}$ & $\begin{array}{l}\text { Ch'odünghak nongŏp } \\
\text { taeyo (Elementary } \\
\text { agriculture) inspection } \\
\text { copy (1909); } \\
\text { Ch'odŭnghak tokbon } \\
\text { (Elementary reading) } \\
\text { inspection copy (1909), } \\
\text { Yuch'i tokbon (1909), } \\
\text { Ch'odŭng igwa (Ele- } \\
\text { mentary science) inspec- } \\
\text { tion copy (1910, in- } \\
\text { cludes an inspection } \\
\text { request form, approved } \\
\text { by the Ministry of Edu- } \\
\text { cation), Pot'onghak } \\
\text { susinsŏ (Ordinary ethics, } \\
\text { presumed to have been } \\
\text { written by Yu Kilchun }\end{array}$ \\
\hline
\end{tabular}




\begin{tabular}{|c|c|c|}
\hline & & or Pak Chŏngtong) \\
\hline Kim Sangyŏn & $\begin{array}{l}\text { Pot'ong kyoyukhak (1908, Ordinary school } \\
\text { pedagogy), Hoesabóp (1909, corporation } \\
\text { law), Chöngson man'guksa (1906, Select- } \\
\text { ed history of the world), Kukgahak (State- } \\
\text { craft), Sangböp ch'ongnon (Introduction to } \\
\text { the commercial code) }\end{array}$ & \\
\hline Yu Sŏngchun & $\begin{array}{l}\text { Pŏphak t'ongnon (1905, Introduction to } \\
\text { law), Sinch'anso pakmurhak (1907, New } \\
\text { textbook of natural history for beginners), } \\
\text { Pot'ong kyogwa taedong yŏksayak (1908, } \\
\text { Survey of Korean history for ordinary } \\
\text { school students }\end{array}$ & \\
\hline Yi Myŏnu & $\begin{array}{l}\text { Sansu kyogwasŏ (1908, Mathematics text- } \\
\text { book) }\end{array}$ & \\
\hline Yi Sangchae & $\begin{array}{l}\text { Ch'odüng saengni wisaenghak taeyo } \\
\text { (1909, Introduction to elementary physiol- } \\
\text { ogy and hygienics) }\end{array}$ & \\
\hline $\begin{array}{l}\text { An } \\
\text { Chonghwa }\end{array}$ & $\begin{array}{l}\text { Ch'odŭng pon'guk yŏksa (1909, Elementary } \\
\text { Korean history), Ch'odŭng man'guk chiri } \\
\text { taeyo (1909, Introduction to elementary } \\
\text { world geography), Ch'odüng taehan chiji } \\
\text { (1907, Elementary geography of Korea), } \\
\text { Ch'odŭng nollihak kyogwasŏ (1907, Elemen- } \\
\text { tary logic textbook), Kukjo inmulji (1909, } \\
\text { Historical figures of Korea), Ch'odŭng taeh- } \\
\text { an chiri (1910, Elementary Korean geogra- } \\
\text { phy), Ch'odŭng wisaenghak kyogwasŏ } \\
\text { (1909, Elementary hygienics textbook), } \\
\text { Ch'odŭng saengni wisaenghak taeyo (1909, } \\
\text { Introduction to elementary physiology and } \\
\text { hygienics), Ch'odŭng susin kyogwasŏ (1910, } \\
\text { Elementary ethics textbook) }\end{array}$ & \\
\hline $\begin{array}{l}\text { Chang } \\
\text { Chiyŏn }\end{array}$ & $\begin{array}{l}\text { Sangŏphak (1907, Commerce), Aegŭp } \\
\text { kŭnseesa (1905, Contemporary history of } \\
\text { Egypt), Yöja tokbon (1908, Women's read- } \\
\text { ing book), Taehan sinjhiji (1907, New } \\
\text { geography of Korea), Sŏsa kŏn'gukji } \\
\text { (1907, Wilhelm Tell), Chópmok sinbŏp } \\
\text { (1909, New grafting techniques), Man'guk } \\
\text { samul kiwŏn yŏksa (1909, History of the } \\
\text { origin of all things in the world) }\end{array}$ & \\
\hline
\end{tabular}


A Hwangsŏng sinmun article from July 23, 1908 remarked, "Yu Kilchun, the director of the Young Korean Academy, took pains to compile a textbook for children and requested the Ministry of Education for inspection of the textbook. But it did not receive the Ministry's approval, and the copies of the textbook were recalled, resulting in a loss of over 6,000 won." 21 At around the same time, the Taehan maeil sinbo (The Korea Daily News) also published a similar article: "Yu Kilchun, the director of the Young Korean Academy compiled a textbook for children, but the Ministry of Education did not approve its publication. Therefore the parts that did not pass the inspection will be revised, and the textbook will be resubmitted for a second inspection from the Ministry of Education." 22

Both articles mentioned that $\mathrm{Yu}$ compiled a textbook for children, which was not published as it did not pass the Ministry of Education's inspection, resulting in monetary loss for the Academy, and that the textbook would be revised to request a second inspection. The articles did not clarify the specific title and only described that it was a textbook that $\mathrm{Yu}$ compiled. But it seems highly likely that the textbook in the articles was Pak Chŏngtong's Yuch'i tokbon. Pak, who had served as the chief editor of the Young Korean Academy at the time, was listed as the author of Yuch 'i tokbon. However, it is difficult to say that he wrote and compiled all the textbooks, and, as mentioned in the Hwangsong sinmun article above, it is highly likely that $\mathrm{Yu}$ had authored textbooks for reading and ethics.

Then why was Yuch'i tokbon unable to pass the inspection? To answer this question, we need to examine the Regulations for Textbook Inspection and the actual inspection process involving the application of the regulations. The inspection copy of Yuch'i tokbon contains evidences of

21 "Pulhŏ kŏmjŏng" (Inspection unapproved), miscellaneous news, Hwangsŏng sinmun, July 23, 1908.

22 "Kyogwasŏ kaejŏng" (Textbook revision), miscellaneous news, Taehan maeil sinbo, July 25, 1908. 
inspection and censorship conducted by the Ministry of Education that it is possible to explore the specifics of the inspection process. The inspection copy has bibliographic information, including the names of the writer, publisher, and censor (inspector), as well as marks created during the inspection and censorship process, such as pokja (a piece of print type placed upside down to mark the place of a syllable block or character that had been unavailable at the time of printing), cross-outs, deletions, revisions, and censor's stamps.

These traces of inspection and censorship were also found in other inspection copies of textbooks. Textbooks for subjects such as ethics, history, and Korean language (reading), contained more materials that encouraged nationalism and patriotism compared to textbooks for other subjects, as warned by the Japanese Residency-General of Korea. As a result, Yuch'i tokbon, Pot'onghak susinsŏ (Ordinary ethics), and Ch'odüng pon'guk yŏk(yak)sa (Elementary survey of Korean history), in particular, were marked with substantial amounts of notes and recommendations for revisions and deletions.

Among these textbooks, an examination of Yuch'i tokbon shows the cover of the inspection copy of the textbook displayed the title of the textbook and was stamped with "copy for inspection." The following table was printed on the back of the cover.

As noted in Table 3, Yuch'i tokbon had been petitioned for inspection on January 25, 1909. Written in han'gŭl, the textbook was composed of three volumes, and it had been written for private school students. The author had been recorded as Pak Chŏngtong, the chief editor of the Young Korean Academy, and the person in charge of publishing was Hong Kichu. ${ }^{23}$ The

23 Hong Kichu, the head of Tongmun'gwan who was in charge of publishing, worked as an administrative official at Posŏng College (Posŏng Chŏnmun Hakkyo) for about two years from 1906. Starting in April 1908, he served as the vice principal of Sujin School (Sujin Hakkyo), founded by the Young Korean Academy, and as the vice principal of Yunghŭi School (Yunghŭi Hakkyo) from September of the same year. In October 1908, he became the head of Tongmun'gwan. 
Table 3. Yuch'i tokbon Inspection Request Form

\begin{tabular}{|c|c|c|c|c|c|c|c|c|c|}
\hline $\begin{array}{l}\text { Deci- } \\
\text { sion } \\
\text { 決定 }\end{array}$ & $\begin{array}{l}\text { Cen- } \\
\text { sor } \\
\text { 査 } \\
\text { 閱 } \\
\text { 員 }\end{array}$ & $\begin{array}{c}\text { Name of } \\
\text { Publisher } \\
\text { 發 } \\
\text { 行 } \\
\text { 者 } \\
\text { 姓 } \\
\text { 名 }\end{array}$ & $\begin{array}{c}\text { Name } \\
\text { of Au- } \\
\text { thor } \\
\text { 著 } \\
\text { 譯 } \\
\text { 者 } \\
\text { 姓 } \\
\text { 名 }\end{array}$ & $\begin{array}{c}\text { Date } \\
\text { of } \\
\text { Compi- } \\
\text { lation } \\
\text { and } \\
\text { Publica- } \\
\text { tion } \\
\text { 版 } \\
\text { 數 } \\
\text { 及 } \\
\text { 發 } \\
\text { 行 } \\
\text { 年 } \\
\text { 月 } \\
\text { 日 }\end{array}$ & $\begin{array}{l}\text { Us- } \\
\text { age } \\
\text { 用 } \\
\text { 途 } \\
\text { 의 } \\
\text { 區 } \\
\text { 別 }\end{array}$ & 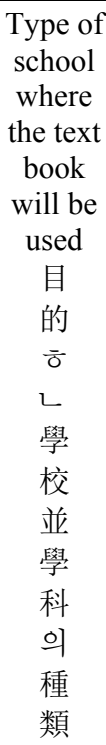 & $\begin{array}{c}\text { Num } \\
\text { ber } \\
\text { of } \\
\text { vol- } \\
\text { umes } \\
\text { and } \\
\text { price } \\
\text { 冊 } \\
\text { 數 } \\
\text { 及 } \\
\text { 定 } \\
\text { 價 }\end{array}$ & $\begin{array}{l}\text { Title } \\
\text { of } \\
\text { text- } \\
\text { book } \\
\text { 圖 } \\
\text { 書 } \\
\text { 의 } \\
\text { 名 } \\
\text { 稱 }\end{array}$ & $\begin{array}{c}\text { Sub- } \\
\text { mission } \\
\text { of orig- } \\
\text { inal } \\
\text { text- } \\
\text { book } \\
\text { 願 } \\
\text { 書 } \\
\text { 接 } \\
\text { 受 }\end{array}$ \\
\hline & $\begin{array}{l}\text { Cen- } \\
\text { sor } \\
\text { 査 } \\
\text { 閱 } \\
\text { 員 }\end{array}$ & $\begin{array}{c}\text { Name of } \\
\text { Publisher } \\
\text { 發 } \\
\text { 行 } \\
\text { 者 } \\
\text { 姓 } \\
\text { 名 }\end{array}$ & $\begin{array}{c}\text { Name } \\
\text { of Au- } \\
\text { thor } \\
\text { 著 } \\
\text { 譯 } \\
\text { 者 } \\
\text { 姓 } \\
\text { 名 }\end{array}$ & $\begin{array}{c}\text { Date } \\
\text { of } \\
\text { Compi- } \\
\text { lation } \\
\text { and } \\
\text { Publica- } \\
\text { tion } \\
\text { 版 } \\
\text { 數 } \\
\text { 及 } \\
\text { 發 } \\
\text { 行 } \\
\text { 年 } \\
\text { 月 } \\
\text { 日 }\end{array}$ & $\begin{array}{l}\text { Us- } \\
\text { age } \\
\text { 用 } \\
\text { 途 } \\
\text { 의 } \\
\text { 區 } \\
\text { 別 }\end{array}$ & $\begin{array}{c}\text { Type } \\
\text { of } \\
\text { school } \\
\text { where } \\
\text { the text } \\
\text { book } \\
\text { will be } \\
\text { used } \\
\text { 目 } \\
\text { 的 } \\
\text { 흘 } \\
\text { 늘 } \\
\text { 學 } \\
\text { 校 } \\
\text { 並 } \\
\text { 學 } \\
\text { 科 } \\
\text { 의 } \\
\text { 種 } \\
\text { 類 }\end{array}$ & $\begin{array}{c}\text { Num } \\
\text { ber } \\
\text { of } \\
\text { vol- } \\
\text { umes } \\
\text { and } \\
\text { price } \\
\text { 冊 } \\
\text { 數 } \\
\text { 及 } \\
\text { 定 } \\
\text { 價 }\end{array}$ & $\begin{array}{l}\text { Title } \\
\text { of } \\
\text { text- } \\
\text { book } \\
\text { 圖 } \\
\text { 書 } \\
\text { 의 } \\
\text { 名 } \\
\text { 稱 }\end{array}$ & $\begin{array}{c}\text { Sub- } \\
\text { mission } \\
\text { of orig- } \\
\text { inal } \\
\text { text- } \\
\text { book } \\
\text { 願 } \\
\text { 書 } \\
\text { 接 } \\
\text { 受 }\end{array}$ \\
\hline
\end{tabular}




\begin{tabular}{|c|c|c|c|c|c|c|c|c|}
\hline $\begin{array}{c}\text { Date } \\
\text { in the } \\
\text { Yung } \\
\text { hŭi } \\
\text { Era } \\
\text { 隆 } \\
\text { 熙 } \\
\text { 年 } \\
\text { 月 } \\
\text { 日 }\end{array}$ & $\begin{array}{c}\text { main } \\
\text { inspec } \\
\text { spec- } \\
\text { tor } \\
\text { 小 } \\
\text { 田 } \\
\text { 主査 } \\
\text { Uemu } \\
\text { ra } \\
\text { 上 } \\
\text { 村 } \\
\text { Hyŏn } \\
\text { Hŏn } \\
\text { 玄 } \\
\text { 櫶 }\end{array}$ & $\begin{array}{c}\text { Hong } \\
\text { Kichu, } \\
\text { Head of } \\
\text { Tong- } \\
\text { mun'gwan } \\
\text { in Su- } \\
\text { dong, } \\
\text { Chung-bu } \\
\text { 中 } \\
\text { 部 } \\
\text { 壽 } \\
\text { 洞 } \\
\text { 同 } \\
\text { 文 } \\
\text { 館 } \\
\text { 長 } \\
\text { 洪 } \\
\text { 箕 } \\
\text { 周 }\end{array}$ & $\begin{array}{c}\text { Pak } \\
\text { Chŏng- } \\
\text { tong, } \\
\text { Chief } \\
\text { editor } \\
\text { of the } \\
\text { Young } \\
\text { Korean } \\
\text { Acad- } \\
\text { emy in } \\
\text { Su- } \\
\text { dong, } \\
\text { Chung- } \\
\text { bu } \\
\text { 中 } \\
\text { 部 } \\
\text { 壽 } \\
\text { 洞 } \\
\text { 興 } \\
\text { 士 } \\
\text { 團 } \\
\text { 編 } \\
\text { 輯 } \\
\text { 部 } \\
\text { 長 } \\
\text { 朴 } \\
\text { 晶 } \\
\text { 東 }\end{array}$ & $\begin{array}{c}\text { For } \\
\text { stu- } \\
\text { dents } \\
\text { 學 } \\
\text { 徒 } \\
\text { 用 }\end{array}$ & $\begin{array}{c}\text { Korean } \\
\text { lan- } \\
\text { guage } \\
\text { classes } \\
\text { at pri- } \\
\text { vate } \\
\text { elemen } \\
\text { men- } \\
\text { tary } \\
\text { school } \\
\text { 私 } \\
\text { 立 } \\
\text { 學 } \\
\text { 校 } \\
\text { 初 } \\
\text { 等 } \\
\text { 敎 } \\
\text { 育 } \\
\text { 國 } \\
\text { 語 } \\
\text { 科 }\end{array}$ & $\begin{array}{c}\text { One } \\
\text { set, } \\
\text { three } \\
\text { vol- } \\
\text { umes } \\
\text { 一 } \\
\text { 帙 } \\
\text { 三 } \\
\text { 冊 } \\
48 \\
\text { chŏn } \\
\text { 四 } \\
\text { 十 } \\
\text { 八 } \\
\text { 錢 }\end{array}$ & $\begin{array}{c}\text { Read- } \\
\text { ing } \\
\text { for } \\
\text { Chil- } \\
\text { dren } \\
\text { 幼 } \\
\text { 穉 } \\
\text { 讀 } \\
\text { 本 }\end{array}$ & $\begin{array}{c}\text { Janu- } \\
\text { ary } 25 \\
\text { in the } \\
\text { third } \\
\text { year of } \\
\text { the } \\
\text { Yungh } \\
\text { ŭi Era } \\
\text { 隆 } \\
\text { 熙 } \\
\text { 三 } \\
\text { 年 } \\
\text { 一 } \\
\text { 月 } \\
\text { 二 } \\
\text { 十 } \\
\text { 五 } \\
\text { 日 }\end{array}$ \\
\hline
\end{tabular}

names to note on the inspection request form are the names of censors who were in charge of the inspection and censorship process. There were three censors: two were Japanese, Oda (小田) and Uemura (上村), and one was Korean, Hyŏn Hŏn (玄櫶). Most notes and recommendations for revisions were written in Japanese, and the censor's stamp next to them belonged to a Japanese censor. Therefore it seems safe to assume that Japanese censors were in charge of overall inspection, and a Korean censor aided them by explaining the contents in Korean, as the Japanese censors probably had difficulty reading in Korean. 
Oda, who was in charge of the inspection of Yuch'i tokbon, is presumed to be Oda Shogo, an official of the Ministry of Education. After graduating from Tokyo Imperial University with a major in history, he began his career in education as a part-time teacher at a school of education in Nagano Prefecture in 1899. He served as a teacher at Hagi Middle School in Yamaguchi Prefecture, the principal of Unebi Middle School in Nara Prefecture, and a professor at Daiichi High School. In November 1908, Oda moved to Korea to succeed Mitsuchi Chuzo as the secretary to the Ministry of Education. In October 1910, he became the head of the editing department under the Japanese Governor-General of Korea. He contributed to the establishment and execution of colonial education policies in colonial Korea, and also played a pivotal role in the compilation process of new textbooks for colonial education after the GovernmentGeneral's promulgation of the First and Second Chosŏn Education Decrees. Moreover, he aided in the founding of Keijo Imperial University in Seoul, taught and lectured on Korean history at the university as a professor afterwards, and shaped the colonial view of history through active participation in academic forums on Korean history and in the Korean History Compilation Committee. ${ }^{24}$

Oda Shogo's activities concerning colonial education began with textbook inspection during the protectorate period, as shown in copies of textbooks for inspection. An in-depth analysis of recommendations for revisions and deletions in the inspection copies will reveal the colonial education that he and the Japanese Residency-General of Korea pursued. Also his involvement with textbook publication from the protectorate period to the colonial period confirms that the textbook inspection and censorship system began to be established at this time and continued into the colonial period. The second Japanese censor, Uemura, also seems to be Uemura Masaki (上邨正己, 上村正己), another official at the Ministry

24 Choe Hyechu, 2010, “Oda Shogo-ŭi kyogwasŏ p’yŏnch'an hwaldong-gwa Chosŏnsa insik" (Oda Shogo's involvement in textbook compilation and awareness of the Korean history), Tongbukayŏksa nonch'ong 27, 279-293. 
of Education. $^{25}$

The Korean censor Hyŏn Hŏn graduated from Kwallip Irŏhakkyo (Japanese Language Governmental School) in January 1905 and became an assistant teacher at Oegugŏ Hakkyo (School of Foreign Languages) in February 1905. In August 1906, he was invited to teach Korean language at Shimane Prefecture Commercial School and then became a professor of Hansŏng Girls' High School in 1907. In August 1907, he began to serve as a translator at the Ministry of Education, and was promoted to the position of compiler at the Ministry in March $1909 .^{26}$ Therefore in January 1909, when Yuch'i tokbon was submitted for inspection, Hyŏn Hŏn took part in the inspection process as a compiler of the Ministry of Education along with the Japanese censors.

This shows that the people who had actually been in charge of textbook inspection around this time were Japanese and Korean officials at the Ministry of Education. The Residency-General established the Textbook Compilation and Inspection Committee within the Ministry of Education. In 1909, the members of the committee included Kumamoto Shigekichi (隈本繁吉, secretary of the Ministry of Education), Yi Mankyu (secretarial official of the Ministry of Education), and Uemura Masaki (上村正己, administrative official of the Ministry of Education). Interim members of the committee were Takahashi Toru (高橋亨, superintendent of Hansŏng High School), Masudo Tsurukichi (增戶鶴吉, superintendent of Hansŏng

\section{Ibid., 285.}

26 As a teacher, he started teaching at Hansŏng Girls' High School Teacher in January 1911, and then at Kyŏngsŏng Ordinary High School and Kyŏngsŏng Girls' Ordinary High School in November of the same year. In February 1921, he became an inspector (sihakgwan) at the Bureau of Education (Hakmuguk) under the Japanese Government-General of Korea. In July of the same year, he also began to serve as an official in charge of textbooks (p'yŏnsugwan) under the Japanese GovernmentGeneral of Korea. In 1931, he was promoted to the position of parliamentary councilor of Gangwon-do (Kangwŏn-do) and retired in March 1934. In April of the same year, he was named as a councilor of the Central Advisory Council of the Japanese Government-General of Korea until 1935. 
Normal School), Munakata (宗像鴨四郎, Hansŏng School of Foreign Languages), Saito Kanji (齋藤欽二, professor of Hansŏng Normal School), Matsumoto Muneharu (松本宗治, professor of Normal High School), Yamauchi (山內愛助, professor of Hansŏng High School), Kojima Kenzaburo (兒島元三郎, professor of Hansŏng High School), and Fukushima (福島百藏, professor of Hansŏng High School). ${ }^{27}$ Among the members of the inspection committee, Uemura participated as a censor in the inspection process of Yuch'i tokbon, and Fukushima was also listed as a censor who oversaw the inspection of Ch'odŭnghak nongŏp taeyo (Elementary Agriculture).

Ueda, who appeared as a censor for the inspection of Ch'odŭnghak tokbon (Elementary reading), was not included in the list of members of the inspection committee, but he was also a compiler at the Ministry of Education. He graduated with a degree in French from Tokyo Imperial University and worked as a translator for the Ministry of War of Japan. After studying in the United States from 1904 to 1907, he returned to serve as a part-time official of the Ministry of Education in Korea. Later, he became a teacher at Kyŏngsŏng High School and an editorial officer at the Japanese Government-General of Korea. ${ }^{28}$

There are pokja, deletions, and revisions in Yuch'i tokbon, which are generally stamped with the censor's seal. Requests for revisions and deletions have been supplemented with reasons for such changes or revised sentences as a side note or on separate strips of papers. Most notes on revisions were simple, involving typos, spelling, or notations. Although there are a number of recommendations for revisions and deletions regarding contents, an in-depth analysis of the recommendations will take

27 Abe Hiroshi ed., 1991, Ilbon singminji kyoyuk chŏngch'aek saryojipsŏng (Chosŏn p'yön) ch'ongmongnok, haeje, saegin(Sourcebook of education policies in Japanese colonies: Korea, table of contents, abstracts, and index), Ch'ŏnggyesǒsa.

28 Chŏng Kŭnsik, 2007, "Kuhanmal Ilbonin-ŭi Chosŏnŏ kyoyuk-gwa t'ongyŏk kyŏngch'al-ŭi hyŏngsŏng" (Japanese Korean language education and the formation of translation police during 1872-1910), Han'guk munhwa yŏn'gu32, 44-45. 
time as some strips of papers with notes have been lost and some characters are difficult to decipher.

Another interesting aspect of the inspection copy of Yuch'i tokbon is that the textbook demonstrates evidence of two processes of inspection. As mentioned in one of the newspaper articles above, Yu Kilchun submitted a copy of Yuch'i tokbon to the Ministry of Education for inspection but failed to receive permission to print it. It seems that he submitted a second version, ${ }^{29}$ as pages of the inspection copy with recommendations for revisions are attached with pieces of paper that contain revised texts, which are also littered with censors' notes for revisions. Despite the two inspection processes, Yuch'i tokbon was never published and used as a textbook due to its inability to meet the Ministry's standards. ${ }^{30}$

Ch'odünghak nongŏp taeyo seems to have been another textbook that did not pass the inspection, as there is no textbook of the same title that we know of today. The author of this textbook was also Pak Chŏngtong, and the person in charge of publishing is recorded as Hong Kichu from T'ongmun'gwan. The censor is marked as Fukushima (福島), speculated to be Fukushima (福島百藏), one of the members of the inspection committee. Fukushima was an expert in the field of agriculture who served as a professor at Hansŏng High School and coauthored Sinch'an nongŏpkyogwasŏ (New textbook on agriculture), ${ }^{31}$ which explains his participation in the inspection of Ch'odŭnghak nongŏp taeyo. However, the censor's stamp imprinted next to the recommendations for revisions of texts in the inspection copy belonged to Oda Shogo, the secretary of the Ministry of Education, just as in Yuch'i tokbon, which implies that Oda made the final decisions in the textbook inspection process.

29 "Pulhŏ kŏmjŏng," miscellaneous news, Hwangsŏng sinmun, July 23, 1908.

30 Although it is beyond the scope of this article, following research on this textbook will closely examine the contents in order to determine the precise reasons for rejection for publication.

31 Kim Ponghŭi, 1999, Han'guk kaehwagi sŏjŏk munhwa yŏn'gu (A study on the book culture in the enlightenment period in Korea), Ihwa Yŏja Taehakkyo Ch'ulp'anbu, 275. 
The inspection copy of Pot'onghak susinsŏ (Ordinary Ethics) contained the most amount of censors' recommendations for revisions and deletions, and it seems to have been resubmitted several times for inspection afterwards. In the inspection copy of this textbook, no record of the author, publisher, or the censor in charge of inspection can be found. The year in which the textbook was authored also does not appear on the textbook. However, a name card with the name Kim Sangch'ŏn is attached to the very first page of the inspection copy. There were records that indicated that Kim was a proprietor of Tongmun'gwan, ${ }^{32}$ and therefore it may be safe to assume that Pot'onghak susinso was another textbook that had been compiled by the Young Korean Academy and published by Tongmun'gwan.

This possibility carries considerable weight since the table of contents and texts of the inspection copy of Pot'onghak susinsŏ and Ch'odüng susin (Elementary Ethics), an ethics textbook for private school students authored by Pak Chŏngtong in 1909, are rather similar. Ch'odŭng susin underwent inspection of the Ministry of Education in April 1909 and was published by Tongmun'gwan on July 12 of the same year. ${ }^{33}$ A comparison of the table of contents and narrative texts reveals that the two textbooks share a considerable amount of similarities.

Despite the similarities, however, these were two different textbooks. Pot'onghak susinsŏ consists of four volumes, one for each grade level from first grade to fourth grade. It is also lengthier than Ch'odŭng susin. In addition, while Ch'odŭng susin was written in Korean mixed script with Chinese characters, the first volume of Pot'onghak susinsŏ for first graders was written only in han'gŭl, and the rest were written in mixed script. The time of publication for these two textbooks also differs. Ch'odŭng susin passed the inspection and was published in 1909. ${ }^{34}$

32 Ibid., 88.

33 An Chonghwa, Pak Chŏngtong et al., 2011, Kŭndae sushin kyokwasŏ 1 (Modern ethics textbook 1), trans. Hŏ Chaeyŏng et al., Somyŏng Ch’ulp'an, 136-174.

34 Ibid., 136-174. 
Pot'onghak susinsŏ, on the other hand, is speculated to have been submitted to the Ministry of Education for inspection immediately before or after the signing of the annexation treaty in August 1910, as the censor recommended revising ponjo (本朝, this dynasty) to Yijo (李朝, Yi dynasty), aguk (我國, our country) and pon'guk (本國, this country) to Chosŏn, and Japan to naeji (內地, inner land) in the section for general revision recommendations. It seems that this copy had been submitted for inspection before Japan's annexation of Korea, and the censors demanded revisions for editing and teaching after the annexation according to the "List of Corrections of Expressions and Cautions for Teaching Using Ordinary School Textbooks Compiled by the Former Ministry of Education and Textbooks Inspected and Approved by the Former Ministry of Education" (1910, hereafter "List of Corrections"). The "List of Corrections" feature information on handling the contents of textbook and teaching materials, such as "terms regarding the names of countries: aguk (我國, our country) and ahan (我韓, our Korea) should be referred to as Chosŏn or peninsula. Japan should be changed to aguk." The recommendations for revisions in the inspection copy of Pot'onghak susinsŏ seem to have followed the rules from the "List of Corrections."

Other textbooks, such as Ch'odüng pon'guk chiri (Elementary Korean geography) and Ch'odüng (hak) igwa (Elementary Science), passed the inspection and were published. These two textbooks did not seem to contain problematic materials, as only revisions of simple expressions and partial deletions were recommended. For these two textbooks, further study is required to compare the inspection copies and the published copies to explore how the censors' recommendations for revisions were reflected in the final version.

\section{Conclusion}

This paper explored the establishment of the textbook inspection and censorship system during the protectorate period, and briefly examined 
the bibliographic and other information included in the newly discovered inspection copies of textbooks that had been authored or compiled by people with connections to the Young Korean Academy. The Young Korean Academy, founded and managed by Yu Kilchun, aimed to proliferate modern education in Korea and actively engaged in projects to foster teaching professionals and to author and compile textbooks. In 1908, the Japanese Residency-General of Korea inspected publicly and privately published textbooks through the Regulations on Textbook Inspection. As a result, the Young Korean Academy petitioned to have the textbooks compiled by the academy or people who had connections to the academy inspected before publication. The inspection copies contained names of Japanese and Korean censors in charge of inspection who were affiliated with the Ministry of Education, as well as evidence of inspection, such as recommendations for revision, censor's stamp, notes for deletion in the text. Some of the textbooks that had been submitted for inspection passed the inspection process and were published, but others were unable to receive approval for publication.

These inspection copies of textbooks are important documents that can be used in historical studies, as well as in education studies, media studies, and Korean literature studies. It is necessary to organize and analyze these documents systematically, as these documents can be used for more intensive research on the educational policies and the censorship system in the Protectorate era. A sourcebook of the inspection copies of textbooks, containing summaries of the text as well as inspection and censorship information of each textbook would greatly contribute to the expansion of research on the inspection and censorship system in early modern Korea, which began in the protectorate period.

I would like to conduct further research by organizing the materials and analyzing the inspection copies of textbooks in depth. This research will first involve an analysis of the ways in which the inspection standards, including the legal regulations stipulated in the Regulations on Textbook Inspection and the internal regulations of the censorship agency, were applied in the actual inspection process. An in-depth analysis of the revi- 
sions and deletions recommended by the censors would also help us understand the standards of the Regulations on Textbook Inspection as well as the detailed standards of inspection and censorship.

Second, this research will involve a comparison of the inspection and censorship system in Japan, Taiwan, which had also been annexed by Japan, as well as in colonial Korea. It would provide an opportunity to explore the ways in which Japan used the inspection and censorship system in the process of establishing itself as an empire and help us examine the similarities and differences of the inspection and censorship systems in Japan and its colonies.

Lastly, this research will investigate the implications of the inspection and censorship system for today. The system of media censorship and textbook inspection was one of the ways in which the government used to acquire and maintain power and rule effectively not only during the Protectorate and colonial periods, but also in the days after the Korean liberation from the Japanese rule and even in recent years. This means that the inspection and censorship system was a system of controlling ideology, information, and the press that was not exclusive to Japan's "protective" rule and colonial rule. By analyzing the historical instances of inspection and censorship, this research will help us gain an understanding of the reason for the continuance of the control system using censorship, including textbook inspection, to today, and the impact this type of control has on Korean society.

\section{References}

1. Kwanbo (Official gazette)

2. Taehan maeil sinbo (The Korea Daily News)

3. Hwangsŏng sinmun (Capital Gazette)

4. Kiho Hŭnghakhoe wŏlbo (Monthly Bulletin of Capital and Central Region Association for Promotion of Learning)

5. T'onggambu munsŏ (Documents of the Japanese Residency-General 


\section{of Korea)}

6. Inspection copy of Ch'odünghak nongŏp taeyo (Elementary agriculture)

7. Inspection copy of Ch'odŭnghak susinsŏ (Elementary ethics)

8. Ch'odünghak pon'guk (yŏk) yaksa (Elementary survey of Korean history)

9. Ch'odüng pon'guk chiri (Elementary Korean geography)

10. Inspection copy of Yuch 'i tokbon (Reading for children)

11. Inspection copy of Ch'odŭnghak tokbon (Elementary reading)

12. Inspection copy of Ch'odŭnghak igwa (Elementary science)

13. Yu Kilchun chŏnsŏ p'yŏnch'an wiwŏnhoep'yŏn, 1971, Yu Kilchun chŏnsŏ (Collected Works of Yu Kilchun) Vol. 2, Iljogak, 1971)

14. Asea Munhwasa P'yŏnjipbu, 1977, Han'guk kaehwagi kyogwasŏ ch'ongsŏ (Series of Korean textbooks during the enlightenment period) 1-20, Asea Munhwasa.

15. Kang, Yunho. 1973. Kaehwagi-ŭi kyogwayong tosŏ (Textbooks from the enlightenment period), Kyoyuk Ch'ulp'ansa.

16. Ku, Taeyŏl. 1986. Chegukjuŭi-wa ŏllon (Imperialism and the press). Ihwa Yŏja Taehakkyo Ch'ulp'anbu.

17. Yun, Kŏnch'a. 1987. Han'guk kŭndae kyoyuk-ŭi sasang-gwa undong (Ideologies and movements of modern education in Korea). Ch’ŏngsa.

18. Ko, Tŏkhwan. 1988. "Ch'ulp'an-gwa ch'ulp'anbŏp" (Printing and printing laws), Ch'ulp'anhak yŏn'gu 90. Pŏmusa.

19. Ch'oe, Kiyŏng. 1991. Taehan cheguk sigi sinmun yŏn'gu (A study on newspapers during the Korean Empire period).Iljogak.

20. Yun, Pyŏnghŭi. 1991. "Yu Kilchun-ŭi Hŭngsadan unyŏng" (Yu Kilchun's management of the Young Korean Academy). Kuksagwan nonch'ong 23.

21. Abe, Hiroshi, ed. 1991. Ilbon singminji kyoyuk chŏngch'aek saryojipsŏng, Chosŏn p'yŏn: ch'ongmongnok, haeje, saegin(Sourcebook of education policies in Japanese colonies: Korea, table of contents, abstracts, and index). Ch’ŏnggyesǒsa. 
22. Yun, Pyŏnghŭi. 1993. "Taehan cheguk malgi Yu Kilchun-ŭi sasanggwa hwaldong" (Yu Kilchun's ideologies and activities in the late Korean Empire period). PhD. Diss. Sŏgang Taehakkyo.

23. Kim, Ponghŭi. 1999. Han'guk kaehwagi sŏjŏk munhwa yŏn'gu (A study on the book culture in the enlightenment period in Korea), Ihwa Yŏja Taehakkyo Ch'ulp'anbu.

24. Yi, Chungyŏn. 2001. “Ch'aek”-ǔi unmyŏng-Chosŏn-Ilje kangjömgi kümsŏ-üi sahoe sasangsa (The fate of "books"- the social and ideological history of banned books from Chosŏn Korea to the Japanese colonial period). Hyean.

25. Kim, Hyojŏn. 2003. "Kuhanmal kyŏngch'al-ŭi iron-gwa silje” (Theories and realities of the police in late Chosŏn). In'gwŏn-gwa chŏngŭi 325.

26. Chŏng, Kŭnsik. 2003. "Singminjijŏk kŏmyŏl-ŭi yŏksajŏk kiwŏn: 1904-1910" (The historical origin of colonial censorship: 19041910).Sahoe-wa yŏksa64.

27. Ishimatsu, Keiko. 2003. "T'onggambu ch'iha Taehan cheguk-ŭi susin kyogwasŏ, kugŏ tokbon yŏn'gu" (A study on ethics textbook and Korean language reading textbook in the Korean Empire under the Japanese Residency-General). MA thesis. Yonsei University.

28. Ku,Hŭichin, 2004. "Han'guk kŭndae kaehyŏkki-ŭi kyoyungnon-gwa kyoyuk kaep'yŏn" (Education theory and reorganization of education in the enlightenment period in Korea). PhD diss. Seoul National University.

29. Han, Mansu. 2004. "Singminji sidae ch'ulp'an chabon-ŭl t'onghan munhak kŏmyŏl-e taehayŏ" (A study on the Korean literary censorship by publication-capitals in Japanese colonial period). Kugo kungmunhak 131.

30. Chŏng, Kŭnsik. 2005. "Iljeha kŏmyŏl kigu-wa kŏmyŏlgwan-ŭi pyŏndong" (Changes in the censorship agency and censors under the Japanese rule).Taedong munhwa yŏn'gu51

31. Kim, Ch'angnok. 2006. "Iljegangjŏmgi ŏllon, ch'ulp'anbŏpje” (The media and press laws of Korea under the Japanese colonial rule). 
Han'guk munhak yŏn'gu 30. Tongguk Taehakkyo Han'guk Munhak Yŏn'guso.

32. Inaba, Tsugio. 2006. Kuhanmal kyoyuk-gwa Ilbonin(Education in the late Chosŏn Korea and the Japanese people). Onnuri.

33. Yi, Yunmi. 2006. Han'guk-ŭi kŭndae-wa kyoyuk (Modern Korea and education). Munŭmsa.

34. Han, Mansu. 2006. “1930 nyŏndae kŏmyŏl kijun-ŭi kusŏng wŏlli-wa chakdong kije" (The organization principle and function mechanism of the censorship standards in the 1930s). Han'gukŏ munhak yŏn'gu 47.

35. Han, Mansu. 2007. "Singminji sigi kŭndae kisul (ch'ŏldo, t'ongsin)gwa inswaemul kŏmyŏl" (The relationships of modern technologyrailway, communication - and print censorship in colonial Korea). Han'guk munhak yŏn'gu 32.

36. Pak, Kwanghyŏn. 2007. "Kŏmyŏlgwan Nishimura Shintaro-e kwanhan koch'al" (A study on Japanese censor Nishimura Shintaro). Han'guk munhak yŏn'gu 32.

37. Son, Chiyŏn. 2007. "Singminji Chosŏn-esŏŭi kŏmyŏl-ŭi sasang-gwa pangbŏp — kŏmyŏl charyojip kuch'uk kwajŏng-ŭl t'onghayŏ" (Concepts and methods of censorship in colonial Chosŏn through the structural process of compiling censorship documents). Han'guk munhak yŏn'gu 32.

38. Chŏng, Kŭnsik. 2007. "Kuhanmal Ilbonin-ŭi Chosŏnŏ kyoyuk-gwa t'ongyŏk kyŏngch'al-ŭi hyŏngsŏng” (Japanese Korean language education and the formation of translation police during 18721910).Han'guk munhwa yŏn'gu32.

39. Yi, Kyehyŏng. 2008. "Taehan chegukki t'onggambu-ŭi singmin kyoyuk ch^ongch'aek" (Research on the colonial education policies of the Japanese Residency-General of Korea in the Korean Empire). PhD diss. Kookmin University.

40. Kim, Soyoung. 2009. "Taehan chegukki 'kukmin' hyŏngsŏngnongwa t'onghamnon yŏn'gu" (A study on the theory of the formation of "citizens" and the theory of integration in the Korean Empire). 
PhD diss. Korea University.

41. T'ongguk Taehakkyo Munhwa Haksurwŏn Han'guk Munhwa, 2010, Singminji sigi kŏmyŏl-gwa Han'guk munhwa (Censorship and the Korean culture in colonial Korea), Tongguk Taehakkyo Ch'ulp'anbu.

42. Choe, Hyechu. 2010. “Oda Shogo-ŭi kyogwasŏ p'yŏnch'an hwaldong-gwa Chosŏnsa insik" (Oda Shogo's involvement in textbook compilation and awareness of the Korean history). Tongbukayŏksa nonch'ong 27.

43. Kim, Soyoung. 2011. "Hanmal susin kyogwasŏ pŏnyŏk-gwa 'kukmin' hyŏngsŏng" (The translation of ethics textbooks and nationbuilding in Korea). Han'guk kŭnhyŏndaesa yŏn'gu 59.

44. Kŏmyŏl Yŏn'guhoe. 2011.Singminji kŏmyŏl (Censoring Colonies), Somyŏng Ch'ulp'an.

45. An Chonghwa, Pak Chŏngtong et al. 2011.Kŭndae sushin kyokwasŏ 1 (Modern ethics textbook 1).Translated by Hŏ Chaeyŏng et al. Somyŏng Ch'ulp'an.

46. Chang, Sin. 2011. "Han'guk kangjŏm chŏnhu ilje-ŭi ch'ulp'an t'ongje-wa '51 chong 20 manwon punsŏ sagŏn-ŭi chinsang" (The Japanese colonial authorities' control of publication during the Japanese occupational period and the 'Burning incident' of 200,000 books). Yǒksa-wa hyŏnsil 80.

47. Chŏng, Kŭnsik. 2012. "Singminji kŏmyŏl-gwa 'kŏmyŏl p'yojun'Ilbon mit Taeman-gwaŭi pigyo-rŭl t'onghayŏ" (Censorship and 'censorship standards' in colonial Korea - comparative study of Japan, Taiwan, and Korea). Taedong munhwa yŏn'gu 79.

48. Chŏng, Kŭnsik. 2013. "Singminji chŏnsi ch'ejeha-esŏŭi kŏmyŏl-gwa sŏnjŏn, kŭrigo tongwon" (Censorship, propaganda, and mobilization of colonial Korea in wartime), Sanghŏ hakbo 38.

49. Chŏng, Chinsŏk.2014.Kŭkbi Chosŏn ch'ongdokbu-ŭi ŏllon kŏmyŏlgwa t'anap-Ilbon-ŭi ch'imnyak-gwa yŏlgang seryŏk-ŭi ŏllon t'ongje (Confidential the Japanese Government-General of Korea and its censorship and oppression of the press-Japanese invasion and the press control of a powerful nation), Kŏmunikeisyŏn buksŭ. 
50. Song, Minho. 2014. “Taehan cheguk sidae ch'ulp'anbŏp-ŭi chejŏnggwa ch'ulp'an kŏmyŏl-ŭi pŏp-munjajŏk kiwŏn" (The enactment of publishing law and legal-literal origin of publication censorship in the Korean Empire). Han'guk hyŏndae munhak yŏn'gu 43.

51. Ha, Sumi. 2014. "Yu Kilchun-ŭi kyoyuk hwaldong-e nat'anan chŏnt'ong-gwa kŭndae" (Tradition and Modernity in Yu Kilchun's education activities). PhD. Diss. Hongik University.

52. Choe, Tŏksu et al. 2015. Kündae Han'guk-ŭi kaehyŏk kusang-gwa $\mathrm{Yu}$ Kilchun (Reformation ideas in modern Korea and Yu Kilchun). Koryŏ Taehakkyo Ch'ulp'an Munhwawŏn.

53. Chŏng, Kŭnsik et al. 2016. Kŏmyŏl-ŭi cheguk-munhwa-ŭi t'ongjewa chaesaengsan (Empire of censorship - the control and reproduction of culture). P'urŭn yŏksa. 
$<$ Abstract $>$

\section{Textbook Inspection and Censorship in Korea during the Protectorate Period: A Study of Inspection Copies of Textbooks Compiled by the Young Korean Academy}

Kim Soyoung

Established during the Japanese protectorate period, the censorship system lasted throughout the colonial period as well. Therefore it is necessary to examine such censorship system as part of Japan's colonization policy and shed light on the historical and current significance of the censorship system as well as its effects. Recently, copies of textbook provided to the Ministry of Education (Hakbu) for inspection in the protectorate period were newly discovered. Materials that have been inspected by the Japanese Residency-General of Korea-inspection copies of textbooks from the Young Korean Academy (Heungsadan)—were included in the "Collection of new materials related to Yu Kilchun". Using these newly discovered copies of textbooks that had been subject to inspection, this study is the first in a series of research that attempted to examine actual cases of textbook inspection and censorship during the protectorate period and shed light on the historical significance of such activities. This study therefore review legislations on inspection and censorship in this period and studied the early stages in the establishment process of a censorship system in modern Korea.

Secondly, this study attempted to provide a bibliographical analysis of the inspection copies of textbooks from the protectorate period, as these are new documents that had never been analyzed or used in research.

Keywords: Textbook inspection, censorship, Japanese Residency-General of Korea, Heungsadan, Yu Kilchun, Pak Chŏngtong 
<국문초록>

\section{통감부시기 교과서 검정과 검열 -흥사단 검정청원 교과서본을 중심으로-}

김소영 (고려대학교 한국사연구소 연구교수)

근대 한국에서 검열제도의 등장은 일본제국주의의 식민지배 확립 과정에서 통 치의 효율성을 높이기 위해 등장했다. '통감부'시기 시작된 검열제도는 이후 식민 지시기 검열제도로 연속되었다. 이러한 검열제도를 문화, 사상, 정보 통제라는 일 본의 식민지배정책의 일환으로 살펴보고 검열제도의 역사적 의미와 현재적 의미, 영향을 밝히는 것이 필요하다.

최근 '통감부'시기 학부, 실제로는 통감부의 교과서 검정의 실태를 보여주는 훙 사단의 교과서 검정 청원본이 발견되었다. 이 교과서 청원본은 2003년 유길준 후 손들이 고려대학교 박물관에 기증한 '유길준 관련 신자료'에 포함되어 있다. 본 연 구는 새롭게 발굴된 교과서 검정 청원본들을 분석하여 '통감부'시기 교과서 검정 및 검열의 실례와 그 역사적 의미를 밝히고자 하는 일련의 연구 가운데 첫 번째 로, 먼저 일본이 식민통치를 확립하기 위해 언론과 출판을 통제하는 검열정책을 '통감부'시기부터 수립, 전개해 갔다는 사실에 주목하여 그 시기 검정 및 검열 관 련 법령을 검토하여 근대 한국에서 검열 체제의 성립 초기 과정을 살펴보았다.

다음으로 '통감부'시기 교과서 검정 청원본은 그동안 분석되거나 활용된 적이 없는 새로운 자료로, 그 서지학적 분석을 시도했다.

주제어: 교과서검정, 검열, 통감부, 흥사단, 유길준, 박정동 
